\title{
Molecular cytological analysis of alien introgressions in common wheat lines with T. kiharae genetic material
}

\author{
O.A. Orlovskaya ${ }^{1 *}$, N.I. Dubovets ${ }^{1}$, L.A. Solovey ${ }^{1}$, E.B. Bondarevich ${ }^{1}$, I.N. Leonova ${ }^{2}$ \\ ${ }^{1}$ Institute of Genetics and Cytology, National Academy of Sciences of Belarus, Minsk, Belarus \\ ${ }^{2}$ Institute of Cytology and Genetics, SB RAS, Novosibirsk, Russia
}

DOI 10.18699/ICG-PlantGen2019-77

(c) Autors, 2019

*e-mail: O.Orlovskaya@igc.by

\begin{abstract}
Monitoring of the process of the introgression of foreign chromatin in the genome of common wheat is the key to the success of the enrichment of the wheat gene pool of agronomically important genes from related species. In the karyotypes of the hybrid lines derived from crosses between common wheat varieties and T. kiharae, the presence of whole chromosomes of $T$. kiharae (intergenomic substitution 2G/2B), chromosome arm substitutions (centric translocation T2AtS:2AL) and large inserts in the form of intercalary translocations involving homoeologous group 1, 3 and 5 chromosomes of the B and $\mathrm{G}$ genomes using C-banding was found. Molecular markers revealed small introgressed fragments of $T$. kiharae into the genomes of common wheat varieties. The highest frequency of introgressions is shown for chromosomes 1A, 1B, 2A, 5B, and 6A. A high level of cytological stability (meiotic index was $88.18-93.0 \%$ ) was noted for the majority of introgression lines, which provides the formation of functional gametes in a number sufficient for successful reproduction. The exceptions are lines 34-1 and 34-2 with structural rearrangements of the chromosome $5 \mathrm{~B}$, affecting the functioning of the main genes for chromosome synapsis, which results in a significant decrease in meiotic index and negatively affects plant productivity.

Key words: T. aestivum; T. kiharae; wheat introgression lines; genomic structure; cytological stability.
\end{abstract}

\section{Introduction}

T. kiharae ( $\mathrm{A}^{\mathrm{t}} \mathrm{A}^{\mathrm{t}} \mathrm{GGDD}, 2 n=42$ ) is of interest for the improvement of bread wheat as a source of high grain protein and gluten content and resistance to many diseases (Pukhalskiy, Odintsova, 2007). The use of T. kiharae to improve T. aestivum L. is complicated by the fact that the degree of chromosome homology between their genomes is low, which leads to an imbalance of sets of chromosomes in gametes of the first generations, and, therefore, and to their low fertility. As a result, the elimination of part of the genotypes in subsequent generations occurs, which reduces the range of inherited variety, and the possibility of selection of valuable forms with the required combinations of genes. The aim of this study was to analyze the genomic structure and cytological stability of hybrid forms obtained in crosses of common wheat varieties with T. kiharae.

\section{Materials and methods}

In this study, $\mathrm{F}_{10}$ lines were obtained at the Institute of Genetics and Cytology of NAS of Belarus from three cross combinations of common wheat $T$. aestivum varieties with T. kiharae: line 31 ('Rassvet' $\times$ T. kiharae); lines 19, 20-1, 25-2 (T. kiharae × 'Saratovskaya 29'); lines 28, 34-1, 34-2 (T. kiharae $\times$ 'Festivalnaya'). Cytological slides and the procedure of C-banding were conducted according to the methodology of Badaeva et al. (1994). The slides were analyzed using an Amplival microscope (Carl Zeiss Jena) with apochromatic 100x lens. Selected metaphase plates were photographed using a Leica DC 300 digital video camera. The images were processed using the Adobe Photoshop graphic editor. Genotyping of the lines and parental varieties was performed using SSR markers (WMC, GSM, GSM) mapped in the genome of hexaploid wheat (Somers et al., 2004). The conditions of polymerase chain reaction (PCR) are described in Röder et al. (1998). Separation of PCR fragments was performed on an ABI PRISM 3100 automatic sequencer (Applied Biosystems, USA). The program GenAlEx M. 6.5 (Peakall, Smouse, 2012) was used for processing the results of SSR analysis, for calculation of the number of alleles and the index of genetic diversity H. Microsporogenesis was studied on squash slides. For each cross combinations and parental forms, 30 plates of metaphase I and 50-80 cells of the following stages of meiosis were analyzed: anaphase I and II, metaphase II, tetrads. Statistical analysis of the data was carried out using Microsoft Excel.

\section{Results and discussion}

To identify the T. kiharae genetic material in the genome of the introgression lines, two approaches were used: C-banding and DNA markers with known chromosomal localization. A combination of both methods provides the most objective assessment of the genomic composition of the lines. Analysis of hybrids with the help of C-banding revealed only one event of introgression of the $\mathrm{A}^{\mathrm{t}}$ genome, the centric translocation T2AtS:2AL, which occurs in the disomic state in all plants of line 19. As for the $\mathrm{G}$ genome, the transfer of its chromatin was carried out mainly due to the formation of intercalary translocations, resulting in the formation of recombinant arms of the chromosome containing genetic material from both wheat species. In total, the lines studied revealed four types of translocations: T1BS.1BL-1GL in line 20-1, T3GS-3BS.3BL and T5GS.5GL-5BL in line 25-2 and T5BS.5BL-5GL in lines 34-1 and 34-2, and all these recombinant chromosomes were present in the karyotypes of plants in the disomic state. Only one line (28) showed introgression of the whole chromosome 
Table 1

Chromosome conjugation in metaphase I and the meiotic index in common wheat lines containing T. kiharae genetic material

\begin{tabular}{llll}
\hline Genotype & Bivalents, pcs & Univalents, pcs & Meiotic index \\
\hline 31 & $20.97 \pm 0.03$ & $0.07 \pm 0.06$ & 92.0 \\
19 & $20.9 \pm 0.06$ & $0.2 \pm 0.11$ & 93.0 \\
$20-1$ & $20.93 \pm 0.05$ & $0.13 \pm 0.09$ & 92.0 \\
$25-2$ & $20.77 \pm 0.10$ & $0.47 \pm 0.21$ & 90.91 \\
28 & $20.93 \pm 0.05$ & $0.13 \pm 0.09$ & 88.18 \\
$34-1$ & $20.7 \pm 0.09$ & $0.6 \pm 0.19$ & 77.0 \\
$34-2$ & $20.13 \pm 0.12$ & $1.8 \pm 0.26$ & 55.83 \\
'Rassvet' & $21.0 \pm 0$ & 0 & 99.0 \\
'Saratovskaya 29' & $20.97 \pm 0.03$ & $0.07 \pm 0.06$ & 99.0 \\
'Festivalnaya' & $20.97 \pm 0.03$ & $0.06 \pm 0.06$ & 84.55 \\
T.kiharae & $20.8 \pm 0.09$ & $0.4 \pm 0.18$ & 80.0 \\
\hline
\end{tabular}

from the $G$ genome in the form of intergenomic $2 \mathrm{G}(2 \mathrm{~B}) \mathrm{sub}$ stitution. At the same time, there were no changes in C-band pattern in line 31 compared with the parental wheat variety. This may be associated with a direction of crosses: in this case, the wheat variety served as the maternal component of hybridization.

Molecular analysis of the hybrid lines was carried out using 72 SSR markers, of which 59 revealed polymorphism between the parental wheat varieties and T. kiharae. The number of postulated introgression fragments expected in the analyzed lines varied from four to eight, with the greatest number noted in line 19. No alleles specific to T. kiharae were found in plants of line 31, which is consistent with the results of C-banding.

Using SSR markers, amplification of PCR fragments specific to T. kiharae was found in chromosome 1B (lines 19, 20-1, 25-2 and 28); in the short arm of chromosome 3B (lines 19 and 25-2). Single introgressed fragments in chromosomes 2BL, 6BS and 7BS were detected only in lines 28, 19 and 34-1, respectively. All lines except line 28 showed changes in the long arm of chromosome $5 \mathrm{~B}$. It can be noted that for the lines with introgression of large fragments of $T$. kiharae chromatin, data of microsatellite analysis coincide with the results of $\mathrm{C}$-banding. The results obtained indicate a high frequency of introgression of T. kiharae genetic material to the genomes of the lines studied with a predominance of short fragments detected by molecular markers.

One of the problems with hybrid forms of cereals is disruption of correct progression of meiosis with the formation of univalent and multivalent associations. In this regard, a comparative study of chromosome behavior at different stages of microsporogenesis in hybrid lines and their parental forms was of great interest. Analysis of the metaphase I stage revealed a high level of bivalent chromosome pairing in all $\mathrm{F}_{10}$ hybrid lines (Table 1).

The greatest number of pollen mother cells (PMC) with disorders at this stage of meiosis $(73.3 \%)$ and the maximum number of univalents $(6.0-3.3 \%$ of MCP) was found in line 34-2 containing a pair of aberrant T5BS.5BL-5GL chromosomes, which is probably associated with a change in the expression of the $\mathrm{Ph} 1$ locus caused by structural reorganiza- tions of chromosome 5B. At the same time, the negative effect of introgression of chromatin of chromosome $5 \mathrm{G}$ in the line 34-1 from the same combination of crosses with a similar intercalary translocation is less pronounced: the number of PMCs with disorders is $26.6 \%$, which, however, is 3-4 times higher than the indicator for the lines without introgressions in chromosome 5B. These dissimilarity may be due to different lengths of the translocated alien fragment.

The meiotic index of $F_{10}$ lines isolated from the 'Rassvet' $\times$ T. kiharae and T. kiharae $\times$ 'Saratovskaya 29' cross combination was 6-9\% lower than in the parental wheat varieties, but exceeded the $90 \%$ level. Even in line 25-2, for which in metaphase I of meiosis the weakening of synapsis was noted due to stabilization of structural reorganizations of the karyotype. The lowest meiotic index was pointed out in lines 34-1 and 34-2 from the T. kiharae $\times$ 'Festivalnaya' cross combination (77.0 and $55.83 \%$, respectively). This fact is in good agreement with the data obtained by analysis of the chromosome behavior of these lines at metaphase I and confirms the negative impact of structural rearrangements identified in chromosomes $5 \mathrm{~B}$ in these lines. The impact of the genetic background of the parental wheat variety 'Festivalnaya' on the cytological stability of these lines should not be excluded either. This wheat cultivar has the lowest meiotic index among the wheat varieties included in the hybridization (see Table 1). In general, the data obtained during the analysis of microsporogenesis allow us to conclude that the level of cytological stability achieved in $\mathrm{F}_{10}$ by the majority of the introgression lines ensures the formation of functional gametes in a number sufficient for the successful reproduction of the hybrid material.

\section{Conclusions}

The results of our study indicate that during the stabilization of the karyotypes of hybrids obtained from crossing common wheat varieties with Kiharae wheat, introgression of T. kiharae genetic material into the T. aestivum genome is carried out both as short translocated fragments, detected only with molecular markers, and as whole chromosomes (intergenomic substitutions) and large fragments (centric and intercalary translocations). The conditions for the preservation of alien substitu- 
tions and translocations in the genome of common wheat is a shift in the disomic state, as well as the participation in the formation of chromosomes of orthologous genomes. Under these conditions, there are no significant disturbances of the process of formation of gametes, which ensures the successful reproduction of introgression lines in a number of generations. The exception is introgressions affecting the functioning of the main genes for chromosome synapsis, which results in a significant decrease in meiotic index and negative effects on the productivity of plants.

\section{References}

Badaeva E.D., Badaev N.S., Gill D.S., Filatenko A.A. Intraspecific karyotype divergence in Triticum araraticum (Poaceae). Plant Syst. Evol. 1994;192(1):117-145. DOI 10.1007/BF00985912.

Badaeva E.D., Budashkina E.D., Bilinskaya E.N., Pukhalskiy V.A. Intergenomic chromosome substitutions in wheat interspecific hybrids and their use in the development of a genetic nomenclature of Triti- cum timopheevii chromosomes. Rus. J. Genet. 2010;46:769-785. DOI 10.1134/S102279541007001X.

Peakall R., Smouse R. GenAlEx 6.5: genetic analysis in Excel. Population genetic software for teaching and research - an update. Bioinformatics. 2012;28(19):2537-2539. DOI 10.1093/bioinformatics/ bts460.

Pukhalskiy V.A., Odintsova T.I. Problems of natural and acquired immunity of plants. To the development of ideas of N.I. Vavilov. Information Vestnik VOGIS. 2007;11(3/4):631-649 (In Russian).

Röder M.S., Korzun V., Wendehake K., Plaschke J., Tixier M.H., Leroy P., Ganal M.W. A microsatellite map of wheat. Genetics. 1998; 149:2007-2023.

Somers D.J., Isaac P., Edwards K. A high-density microsatellite consensus map for bread wheat (Triticum aestivum L.). Theor. Appl. Genet. 2004;109(6):1105-1114. DOI 10.1007/s00122-004-1740-7.

Acknowledgements. The work is supported by BRFBR (project No. B18R-028) and RFBR (project No. 18-516-00001).

Conflict of interest. The authors declare no conflict of interest. 